\title{
Spin-Lattice Relaxation in Si Quantum Dots
}

\author{
B. A. Glavin \\ Institute of Semiconductor Physics, Ukrainian National Academy of Sciences, Pr. Nauki 45, Kiev \\ 03028, Ukraine \\ K. W. Kim \\ Department of Electrical and Computer Enginering, North Carolina State University, Raleigh, \\ NC 27695-7911, USA
}

\begin{abstract}
We consider spin-lattice relaxation processes for electrons trapped in lateral Si quantum dots in a [001] inversion layer. Such dots are characterized by strong confinement in the direction perpendicular to the surface and much weaker confinement in the lateral direction. The spin relaxation is assumed to be due to the modulation of electron $g$-factor by the phonon-induced strain, as was shown previously for the shallow donors. The results clearly indicate that the specific valley structure of the ground electron state in Si quantum dots causes strong anisotropy for both the one-phonon and two-phonon spin relaxation rates. In addition, it gives rise to a partial suppression of the twophonon relaxation in comparison to the spin relaxation of donor electrons.
\end{abstract}

Typeset using REVTEX 


\section{INTRODUCTION}

Recently, there is a growing interest in the physics of electron spin due to the enormous potential of spin-based devices. In these so-called "spintronic" devices, information is encoded in the spin state of individual electrons. Numerous concepts ranging from spin analogs of conventional electronic devices, to quantum computers (which utilize the Zeeman doublet of a confined electron as a qubit) 11 have been proposed. Electron spin states relax by scattering with imperfections or elementary excitations such as phonons. Hence, the spin relaxation time is a vital characteristic that determines the potential value of a spin-based device.

In the present paper, we calculate the longitudinal spin-lattice relaxation time $\left(T_{1}\right)$ for electrons confined in Si quantum dots (QDs). Being a relatively light semiconductor, Si is characterized by a weak spin-orbit interaction, which basically determines the strength of spin relaxation. In addition, only a small fraction of the isotopes in natural Si possess a nonzero nuclear magnetic moment. As a result, the electron-nuclear spin-flip process is expected to be slow. These two properties of Si, along with the tempting possibility of integrating the "quantum" part of a computer with the well-developed Si "classical" electronics, make Si an attractive material for spin devices.

We concentrate on a particular design of QD based on a [001] inversion layer formed at the interface of $\mathrm{Si}$ and $\mathrm{SiO}_{2}$ [see Fig. 1(a)]. The lateral confinement of electrons is assumed to be due to the attractive potential applied to the gate electrode deposited on top of the oxide layer. Alternative QD design based on Si/SiGe heterostructures is also possible. However, the SiGe-based design may face additional complications for quantum computing applications. For example, electron confinement in $\mathrm{Si} / \mathrm{SiGe}$ heterostructures is relatively weak and the penetration of electron wavefunction to the SiGe barriers is inevitable. Since spin-orbit interaction in $\mathrm{Ge}$ is stronger than in $\mathrm{Si}$, these structures are expected to have higher spin relaxation rates.

In our calculations, we take advantage of the results obtained for spin relaxation of 


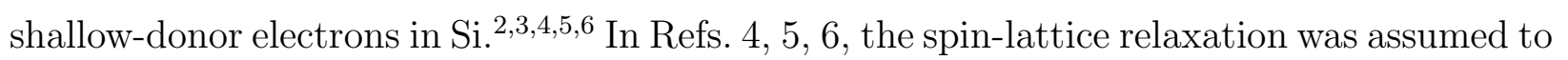
be due to the modulation of electron $g$-factor by the phonon-induced strain. The parameters of the effective Hamiltonian were estimated from microscopic models. Later, they were also determined by measuring the $g$-factor under a static strain. structure of the electron states in lateral QDs, we calculate the corresponding spin relaxation time for both single-phonon and two-phonon processes. Simple analytical expressions are obtained for the two limiting cases, namely, for $T \ll E_{Z}$ and $T \gg E_{Z}$, where $T$ is the temperature in energy units and $E_{Z}$ is the Zeeman doublet splitting energy. The former case is more relevant to the conditions for quantum computation, while the latter is close to the typical conditions of EPR measurements. For the resonance frequency about $50 \mathrm{GHz}$ and low enough temperature, the spin relaxation time is found to be several minutes with the single-phonon process providing the prevailing contribution to relaxation. For elevated temperatures, the two-phonon process becomes significant and the relaxation time can be substantially smaller. We also predict strong anisotropy in the relaxation rate. In particular, for the [001] inversion layer, the spin relaxation is suppressed if the applied magnetic field is parallel to the [001] or [110] directions.

The rest of the paper is organized as follows: In Section II, we introduce the Hamiltonian for spin-lattice relaxation and provide expressions for the single-phonon and two-phonon relaxation rates. In Section III, the asymptotic dependences for different temperatures as well as numerical results are presented. Finally, Section IV is devoted to the discussion of the obtained results along with other potential mechanisms of spin-lattice relaxation.

\section{EFFECTIVE HAMILTONIAN FOR SPIN-LATTICE RELAXATION}

For crystals which possess inversion symmetry like Si, there is no analog of conventional deformation potential for the spin-flip process, which is often called Van Vleck cancellation. $\mathrm{G}$ This is a direct result of the requirement for the Hamiltonian to be invariant under a generalized inversion transformation $C=J K$, where $J$ and $K$ are spatial and time inversion 
operators. In Refs. 6 , 5, 6, the following effective $C$-invariant Hamiltonian describing the modulation of electron $g$-factor by strain has been proposed:

$$
H_{g}=A_{i j k l} u_{i j} B_{k} \sigma_{l},
$$

where $A_{i j k l}$ are the coefficients, $u_{i j}$ is the strain tensor, $B_{k}$ are the components of the magnetic field, and $\sigma_{l}$ are the Pauli matrices; here and below we assume summation over the repeated indices. $H_{g}$ is written in the basis of the Bloch functions corresponding to the bottom of the conduction band and can be used for calculations of the spin transitions for the electron states described within the effective mass approximation. Nonzero coefficients $A$ are determined by the symmetry of the crystal using the method of invariants. for the $\Delta$ point of the Brillouin band in a diamond-like crystal that corresponds to the conduction band of $\mathrm{Si}$, there are eight invariants and the Hamiltonian for a [001] valley can be written as:包

$$
\begin{array}{r}
H_{g}^{[001]}=\frac{1}{2} \mu_{B}\left(A_{1} \sigma_{z} B_{z}\left(u_{x x}+u_{y y}\right)+A_{2} \sigma_{z} B_{z} u_{z z}+\right. \\
A_{3} \sigma_{z}\left(B_{x} u_{x z}+B_{y} u_{y z}\right)+A_{4}\left(\sigma_{x} B_{x}+\sigma_{y} B_{y}\right) u_{z z}+ \\
A_{5}\left(\sigma_{x} B_{x}+\sigma_{y} B_{y}\right)\left(u_{x x}+u_{y y}\right)+A_{6}\left(\sigma_{x} B_{y}+\sigma_{y} B_{x}\right) u_{x y}+ \\
\left.A_{7}\left(\sigma_{x} u_{x z}+\sigma_{y} u_{y z}\right) B_{z}+A_{8}\left(\sigma_{x} B_{x}-\sigma_{y} B_{y}\right)\left(u_{x x}-u_{y y}\right)\right) .
\end{array}
$$

Here, we introduced the factor $\mu_{B} / 2$ for convenience, $\mu_{B}$ being the Bohr magneton. The absolute values of the coefficients $A$ can be determined in principle by using a many-band effective-mass expansion of the electron wavefunction in a uniform magnetic field, similar to that used in Ref. 5. In practice, this cannot be accomplished since the required momentum matrix elements are unknown. However, some qualitative considerations are possible. Since the terms of the expression for $g$-factor contain the energy gaps between the coupled bands in the denominator, coupling of the closest bands is expected to be the strongest. For $\mathrm{Si}$, there is a $\Delta_{2}^{\prime}$ band which is close to the $\Delta_{1}$ conduction band. These bands merge at the $X$ point in the momentum space but are not coupled by either a spin-orbit interaction or momentum operators. Therefore, $\Delta_{1} \leftrightarrow \Delta_{2}^{\prime}$ coupling is not manifested in the effective mass or $g$-factor 
of unstrained Si. However, these bands are coupled by the deformation potential. From the character tables of the $\Delta$ point and the corresponding invariants, it is easy to conclude that this coupling is realized by the deformation potential term proportional to $u_{x y}$. Since $H_{g}$ ccontains only one invariant proportional to $u_{x y}$, it can be concluded that the major contribution to the Hamiltonian is

$$
H_{g}^{[001]}=\frac{1}{2} A \mu_{B}\left(\sigma_{x} B_{y}+\sigma_{y} B_{x}\right) u_{x y}
$$

where we drop the index of the coefficient $A_{6}$. This argument was initially used by Roth. 8

To proceed with the calculation of the longitudinal relaxation time $T_{1}$, we need to describe explicitly the system under consideration as well as the characteristic energy and length scales. As mentioned in the introduction, we consider $\mathrm{Si}$ lateral QDs formed at the $\mathrm{Si} / \mathrm{SiO}_{2}$ interface, where the lateral confinement is due to the gate electrodes. For such a system, the lateral dimension of the $\mathrm{QD} a_{l a t}$ is typically on the order of one hundred $n m$, exceeding considerably the inversion layer thickness $a_{2 D}$. The energy structure of the electron levels is determined by the following parameters: (a) quantization energy in the 2D inversion channel $E_{2 D} \sim \hbar^{2} /\left(2 m_{2 D} a_{2 D}^{2}\right)$; (b) lateral quantization energy $E_{\text {lat }} \sim \hbar^{2} /\left(2 m_{\text {lat }} a_{\text {lat }}^{2}\right)$; (c) intervalley splitting energy between [001] and [001] states, $\Delta$; and (d) Zeeman energy $E_{Z}=\mu_{B} g B$, where $g$ is the effective $g$-factor of the confined electrons depending, in general, on the direction of the magnetic field. Here $m_{2 D}$ and $m_{l a t}$ are the electron effective masses in the direction normal and parallel to the inversion layer, respectively. For the [001] inversion layer, they just correspond to the longitudinal and transverse effective masses of Si. In the following, we assume that the conditions $E_{Z} \ll \Delta, E_{l a t}$ and $E_{l a t} \ll E_{2 D}$ are satisfied. In fact, this is a necessary requirement for spin qubit operation. Using modern technology, $E_{\text {lat }}$ can be made about a $m e V$ or even higher. In contrast, $\Delta$ can be controlled in a much lesser degree, and is roughly proportional to the confining electric field in an inversion layer. Experimentally, $\Delta$ was measured to be up to $1 \mathrm{meV}$ in strong fields (see Ref. 10). In Fig. 1(b), we show schematically the energy levels and the electron transitions under consideration. The numbers "0" and "1" mark the levels of lateral electron confinement. 
The signs + and - denote the valley-split electron states, and "up" and "down" are the spin states. We assume that only the lowest Zeeman doublet can be populated, which means that the temperature $T$ is much less than $\Delta$ and $E_{\text {lat }}$. The longitudinal relaxation time $T_{1}$ is determined as $T_{1}^{-1}=W_{u p-d o w n}+W_{d o w n-u p}$, where $W$ are the probabilities of spin-up to spin-down and spin-down to spin-up transitions. The solid arrows indicate the single-phonon transitions, while the dashed arrows correspond to the two-phonon process. Later in this section we comment on the possible two-phonon processes shown in the figure.

In Refs. 4 and 5, an alternative Hamiltonian to Eq. (3) was considered. It originates from the coupling of the donor singlet and doublet states by an applied magnetic field. It arises due to the different valley structure of these states and the anisotropy of the $g$-factor in the individual valleys. Although this Hamiltonian does not involve $\Delta \leftrightarrow \Delta_{2}^{\prime}$ coupling, its contribution is high because of a very small gap between the singlet and doublet states. However, there is no such mechanism in the [001] lateral QDs, which is probably the main difference with the case of spin relaxation for donor electrons. The reason for that can be easily seen. For the [001] inversion layer, the ground electron state is a combination of [001] and $[00 \overline{1}]$ states:

$$
\Psi_{ \pm}=\chi\left(F_{001}^{ \pm} \psi_{001}+F_{00 \overline{1}}^{ \pm} \psi_{00 \overline{1}}\right)
$$

where we dropped the spin index of wavefunctions. Here $\chi$ is the envelope wavefunction, $\psi_{001}$ and $\psi_{00 \overline{1}}$ are the Bloch functions corresponding to the [001] and [001] valleys, respectively, and $F$ are the coefficients which determine the valley splitting. Particular expressions for $F$ can be found using a microscopic model, for example, that of Sham and Nakayama.11 In our case we do not need explicit expressions for $C$. It is enough to use the fact that Zeeman Hamiltonian has identical forms for the [001] and [001] valleys and its intervalley matrix elements are zero. Therefore, the matrix element between $\Psi_{+}$and $\Psi_{-}$, which is proportional to the overlap between them, is zero because these states are orthogonal.

The Hamiltonian of Eq. (3) is written in the representation where the basis electron wavefunctions correspond to the definite spin projections on the $z$ axis. For calculation 
of $T_{1}$, it must be rewritten by using a representation with the definite spin projection on the direction of the magnetic field. 2 This can be done following the standard procedure of transformation for Pauli matrixes under rotation.13 Finally, we obtain the expression for the one-phonon relaxation rate, $1 / T_{1}^{(1)}$ as:

$$
\frac{1}{T_{1}^{(1)}}=\frac{\pi^{3} A^{2}}{4} \frac{\hbar f^{5}}{g^{2} \rho}\left(1+2 N_{T}\right) \sin ^{2} \theta\left(\cos ^{2} 2 \phi+\cos ^{2} \theta \sin ^{2} 2 \phi\right) \sum_{i} \int d \Omega_{q}^{(i)} \frac{\left(e_{x}^{(i)} n_{y}^{(i)}+e_{y}^{(i)} n_{x}^{(i)}\right)^{2}}{s_{i}^{5}}
$$

In this equation, $g$ is the slightly anisotropic $g$ factor of confined electrons, $f=g \mu_{B} B /(2 \pi \hbar)$ is the resonance frequency, $\theta$ and $\phi$ are the spherical angles of the magnetic field, $\rho$ is the material density, $N_{T}$ is the Planck phonon population for the energies equal to $E_{Z}, \Omega_{q}$ is a solid angle in the phonon wavevector space $\boldsymbol{q}, \boldsymbol{e}$ and $\boldsymbol{n}$ are the phonon polarization vector and the unit vector parallel to $\boldsymbol{q}$, respectively, $s$ is the sound velocity, and the summation is over the acoustic phonon branches. Equation (5) assumes that the phonon wavelength corresponding to the energy $E_{Z}$ is much greater than the lateral dimensions of QD. In this case, the form factor of the electron-phonon interaction is equal to unity and $T_{1}^{(1)}$ does not depend on the particular shape of the lateral confining potential. To check the validity of this approach, we performed calculations of $T_{1}^{(1)}$ assuming parabolic lateral confinement and found that for the lateral level separation of $1 \mathrm{meV}$, the obtained correction is less than $10 \%$ even for $f=50 \mathrm{GHz}$. An additional assumption of the bulk-like phonon spectrum is made for simplicity. This probably introduces a greater error since the lateral QDs are normally situated close to the surface. As shown in Refs. 14, 15, 16, the phonon modes in this case are essentially rebuilt, due to the interference of the incident and reflected phonons as well as the origination of Rayleigh waves, which strongly modify electron-phonon coupling.

Equation (5) predicts strong anisotropy of the relaxation rate. If the magnetic field is parallel to the [001] or [110] directions, $T_{1}^{(1)}$ goes to infinity. This is because we used a single-parameter Hamiltonian of Eq. (3). When rewritten for the basis with the definite spin projection on the direction of the magnetic field, it vanishes for these particular orientations. Of course, in experiments the relaxation for these cases is not expected to be suppressed completely, since the remaining terms of Eq. (2) have non-zero contributions to the relaxation 
rate. However, a significant decrease is expected.

Assuming an isotropic acoustic phonon spectrum, we obtain

$$
\frac{1}{T_{1}^{(1)}}=\frac{2 \pi^{4} A^{2}}{5} \frac{\hbar f^{5}}{g^{2} \rho s_{t}^{5}}\left(1+2 N_{T}\right) \sin ^{2} \theta\left(\cos ^{2} 2 \phi+\cos ^{2} \theta \sin ^{2} 2 \phi\right) .
$$

Here we take into account only TA phonons, which provide the major contribution to the relaxation rate.

Let us now turn to the calculation of the relaxation time due to the two-phonon transitions $T_{1}^{(2)}$. This is a second-order transition where the electron is virtually scattered first to an intermediate state and then to the final state. One of the virtual transitions is accompanied by spin flip, while the other occurs with spin conservation. The probability of a two-phonon transition can be found using the second-order perturbation theory (see, for example, Ref. 17). For the spin relaxation of donor electrons, the intermediate electron state is represented by the excited doublet. In contrast, for the case of [001] lateral QD, the valley-split state cannot serve as an intermediate state. This is because the valley-split states are not coupled by the Hamiltonian of Eq. (2). This can be shown using a argument similar to that applied for the proof of the absence of $g$-factor modulation due to coupling of the valley-split states. Other possible transitions are through the excited states of the lateral confinement. Since the intervalley splitting is controlled by the electron confinement in the $z$ direction rather than in the lateral direction, these transitions are actually possible between the states having the same valley structure [see Fig. 1(b)]. If the phonon wavevector is very small, then the probability of such a transition goes to zero because the overlap functions of "0" and "1" states are orthogonal. To determine the probability of two-phonon transition for a finite phonon wavevector, we need to know the overlap function $\chi$ explicitly. For calculations, we assume parabolic lateral confinement. In this case, the "lateral" electron state is determined by the two quantum numbers, $l_{x}$ and $l_{y}$. The " 0 " state corresponds to $l_{x}=0$, $l_{y}=0$, and there are two degenerate " $1 "$ states with $l_{x}=0, l_{y}=1$, and $l_{x}=1, l_{y}=0$. Using the expressions for the wavefunctions of harmonic oscillator, 13 it is easy to obtain the necessary form-factor $J$ : 


$$
J \equiv \int d x d y d z \chi_{00} \chi_{10} \exp \left(i\left(q_{x} x+q_{y} y+q_{z} z\right)\right)=\frac{1}{\sqrt{2}} \frac{q_{x}}{k} \exp \left(-\frac{q_{x}^{2}+q_{y}^{2}}{4 k^{2}}\right),
$$

where the subscript of $\chi$ represent the $l_{x}$ and $l_{y}$ quantum numbers and $k$ is expressed through the energy gap $\delta$ between "0" and "1" states and the lateral effective mass: $k=$ $\sqrt{m_{\text {lat }} \delta} / \hbar$. Here we take into account that the thickness of the inversion layer is much less than the typical phonon wavelength. In the following, we also assume that the typical phonon wavevector is less than $k$, and drop the exponent in the expression for $J$. Note, that $J$ can be modified due to the diamagnetic influence of the magnetic field as well. In particular, it can lift the degeneracy of "1" states. In our calculations, we do not consider this effect.

We can distinguish three contributions to the two-phonon relaxation rate: (a) due to emission of two phonons, (b) due to absorption of two phonons, and (c) due to phonon scattering. For each of them the rate can be expressed in a uniform manner:

$$
\frac{1}{T_{1}^{(2)}}=34 \pi^{10}\left(\frac{16}{105}\right)^{2} \frac{A^{2} E_{2}^{2} \hbar^{6} f^{13}}{\delta^{4} g^{2} \rho^{2} s_{t}^{14} m_{\text {lat }}^{2}} \sin ^{2} \theta\left(\cos ^{2} 2 \phi+\cos ^{2} \theta \sin ^{2} 2 \phi\right) D_{i} .
$$

Here only the transverse phonons are taken into account, which provide the major contribution to the relaxation rate. We also assume that typical phonon energies are considerably less than $\delta$. The spin-conserving virtual transition is treated within the deformation model, which for the [001] valley provides the interaction $E=E_{1} u_{i i}+E_{2} u_{z z}$, where $E_{1}$ and $E_{2}$ are the deformation potential constants. The coefficients $D$ depend on temperature and they are different for each of the three processes mentioned:

$$
\begin{array}{r}
D_{e m}=\int_{0}^{1} d x x^{5}(1-x)^{5}\left(1+\frac{1}{\exp (x / t)-1}\right)\left(1+\frac{1}{\exp ((1-x) / t)-1}\right), \\
D_{a b}=\int_{0}^{1} d x x^{5}(1-x)^{5} \frac{1}{\exp (x / t)-1} \frac{1}{\exp ((1-x) / t)-1}, \\
D_{\text {scat }}=\frac{20}{17}\left(\int_{0}^{\infty} d x x^{5}(1+x)^{5} \frac{1}{\exp (x / t)-1}\left(1+\frac{1}{\exp ((1+x) / t)-1}\right)+\right. \\
\left.\int_{0}^{\infty} d x x^{5}(x+1)^{5} \frac{1}{\exp ((x+1) / t)-1}\left(1+\frac{1}{\exp (x / t)-1}\right)\right),
\end{array}
$$

where $t=T / E_{Z}$. One can see, that the two-phonon relaxation rate is characterized by the same anisotropy as in the one-phonon relaxation rate. In the following section, we analyze 
the asymptotic dependences of the relaxation rates for different temperature regimes and perform numerical calculations.

\section{RELAXATION RATES FOR LOW AND HIGH TEMPERATURES: NUMERICAL RESULTS}

For the limiting cases of $T \ll E_{Z}$ and $T \gg E_{Z}$, the relaxation rates obey simple power laws as a function of resonance frequency and temperature. In particular, $T_{1}^{(1)} \sim f^{-5}$ in the former case and $T_{1}^{(1)} \sim f^{-4} T^{-1}$ in the latter case. This is similar to the case of donor spin relaxation. main contribution from the two-phonon emission. For $T \gg E_{Z}$, the relaxation is mainly due to the phonon scattering and $T_{1}^{(2)} \sim f^{-2} T^{-11}$. For donor electrons, Roth obtained a different power law. transition in the lowest approximation is equal to unity, in contrast to Eq. (7) for the lateral QD where the form-factor is suppressed for long-wavelength phonons.

In Figs. 2 and 3, we show the results of numerical calculations for one-phonon and twophonon rates. We assume a magnetic field parallel to the [100] direction, $\rho=2329 \mathrm{~kg} / \mathrm{m}^{3}$, the transverse sound velocity $s_{t}=5420 \mathrm{~m} / \mathrm{s}, E_{2}=10 \mathrm{eV}, m_{\text {lat }}=0.19 m_{0}, \delta=2 \mathrm{meV}$, and $g=2$. The coefficient $A$ can be determined by the measurement of the donor $g$-factor in strained Si. With this method, $A=1.32$ was obtained in Ref. 2. The dependence of the spin relaxation rate on the resonance frequency is presented in Fig. 2 for several temperatures. One can see that for $T_{1}^{(1)}$ the transition between the described power dependences at the different temperature regimes is quite fast. This is not the case for $T_{1}^{(2)}$, which is due to the different temperature dependence of phonon absorption, emission, and scattering rates.

In Fig. 3, we plot the relaxation rates as a function of $f$ under the condition $T / E_{Z}=$ const. This is relevant to the case of QD-based qubit, where this ratio must be kept small to ensure initial state preparation of the qubits. We see that under this condition the major contribution is provided by one-phonon scattering. 
Of course, the predicted for small resonance frequencies huge relaxation times hardly can be measured in experiments. This is similar to the case of donor spin relaxation, 2 where actual experiments were undertaken for $f$ about tens of $G H z$.

\section{DISCUSSION}

Let us first summarize the major distinction of the spin-relaxation process for lateral QDs and shallow donors. First, we predict that the relaxation for lateral QDs is more anisotropic than that for donor electrons. In particular, for [001] inversion layer the relaxation rate is suppressed for a magnetic field parallel to the [001] or [110] direction. Second, the twophonon relaxation in lateral QDs is, in general, weaker than for donor electrons and is characterized by different power dependences on the resonance frequency and temperature. Both of these features arise due to the different valley structure of the electron states in lateral QDs in comparison to that of donor electrons.

We have to stress that the first conclusion relies on the model used for calculations, which must be checked by experiments. This is because the values of the coefficients $A_{n}$ in Eq. (2) are determined not only by the energy gaps between the bands, but also by a number of interband matrix elements, which are unknown. Strictly speaking, the experiments with donors can not be considered as a rigorous proof of single-valley Hamiltonian of Eq. (3). In fact, for the donor electrons, eight invariants of Eq. (2) are transformed to three invariants after summation over the valleys. The term measured in Ref. 凤, $\left.H \sim u_{x y}\left(\sigma_{x} B_{y}+\sigma_{y} B_{x}\right)\right)+c p$ where $c p$ stands for cyclic permutations, is obtained from several terms of Eq. (2)), not only from that of Eq. (3)).

There is another mechanism that can modify the spin relaxation in the lateral QDs. This can take place if some quantum levels originated from the longitudinal [001], [001] valleys and the transverse $[100],[\overline{100}],[010],[0 \overline{1} 0]$ valleys come close. In this case, the intervalley coupling can mix these two groups. Mixing of these states and the ground state of the QD by both steady-state and strain-induced contributions of Zeeman Hamiltonian can be possible. 
As a result, both one-phonon and two-phonon relaxation will be modified. According to selfconsistent calculations, 10 such a situation is possible for particular parameters of Si inversion layer.

Finally, it is necessary to take into account alternative forms of the Hamiltonian which also cause spin relaxation. For example, the following Hamiltonian is possible:

$$
H_{s p}=D_{i j k l} \frac{\partial u_{i j}}{\partial r_{k}} \sigma_{l} .
$$

As with $H_{g}$, this Hamiltonian is invariant under inversion $C$. Physically, it describes the splitting of the spin states by nonuniform deformation. A similar relaxation process proportional to the third power of the phonon wavevector, was discussed previously in Ref. 18. We believe that the disagreement between the theoretical calculations of $T_{1}$ based on $H_{g}$ and experimental measurements l $^{2}$ can be partially explained by the contribution of $H_{s p}$. Indeed, it is easy to check that $H_{s p}$ provides the same dependence of the one-phonon rate on frequency and temperature as $H_{g}$. This is because the energy conservation makes the magnetic field and the phonon wavevector proportional to each other. In Ref. Q, the measured one-phonon rate was about two times higher than the calculated one. The two-phonon relaxation was found to obey the predicted temperature dependence, but in contradiction to $H_{g}$, demonstrated no dependence on the magnetic field. Assuming that the contribution of $H_{s p}$ to one-phonon relaxation is about that of $H_{g}$, the contribution of $H_{s p}$ to two-phonon rate in this case can be much greater than that of $H_{g}$ since the typical phonon energy for two-phonon process is much greater than that of Zeeman splitting. Hence, this assumption can explain both the quantitative disagreement obtained for one-phonon relaxation and the qualitative one obtained for two-phonon relaxation. Of course, this idea must be checked experimentally. In particular, important information can be obtained from the measurement of possible spin splitting in nonuniformly strained Si. Consideration of the symmetry properties and the absolute value of such splitting will be provided elsewhere.

Finally, we would like to stress that the electron spin relaxation in QDs is of the same order of magnitude as that obtained for donors. It is much longer than the $T_{1}$ in III-V 
compounds, which proves a good perspective of Si for quantum information processing.

\section{ACKNOWLEDGEMENTS}

The work performed at North Carolina State University was supported by the Office of Naval Research and the Defense Advanced Research Projects Agency. 


\section{REFERENCES}

${ }^{1}$ D. Loss and D. P. DiVincenzo, Phys. Rev. A 57, 120 (1998).

${ }^{2}$ D. K. Wilson and G. Feher, Phys. Rev. 124, 1068 (1961).

${ }^{3}$ A. Honig and E. Stupp, Phys. Rev. 117, 69 (1960).

${ }^{4}$ H. Hasegawa, Phys. Rev. 118, 1523 (1960).

${ }^{5}$ L. Roth, Phys. Rev. 118, 1534 (1960).

${ }^{6}$ L. Roth, in Proc. Intl. Conf. Phys. Semicond. 1960 (Publishing House of the Czechoslovak Academy of Sciences, Prague, 1961), p. 592.

${ }^{7}$ J. H. Van Vleck, Phys. Rev. 57, 426 (1940).

${ }^{8}$ G. L. Bir and G. E. Pikus, Symmetry and Strain-Induced Effects in Semiconductors (Wiley, New York, 1974).

${ }^{9}$ V. I. Sheka, private communication.

10 T. Ando, A. B. Fowler, and F. Stern, Rev. Mod. Phys. 54, 437 (1982).

${ }^{11}$ L. J. Sham and M. Nakayama, Phys. Rev. B 20, 734 (1979).

${ }^{12}$ It is worth noting that such wavefunctions are not the eigenfunctions of the Hamiltonian when spin-lattice coupling is not considered. This is a result of the anisotropy in the electron $g$-factor. For Si, this anisotropy is very weak and is not taken into account.

${ }^{13}$ L. D. Landau and E. M. Lifshitz, Quantum Mechanics: Non-relativistic Theory (Pergamon, Oxford, 1977).

${ }^{14}$ S. M. Badalyan and I. B. Levinson, Fiz. Tverd. Tela 30, 2764 (1988) [Sov. Phys. Solid State 30, 1592 (1988)].

${ }^{15}$ Y. M. Sirenko, K. W. Kim, and M. A. Stroscio, Phys. Rev. B 56, 15770 (1997). 
${ }^{16}$ V. I. Pipa, F. T. Vasko, and V. V. Mitin, J. Appl. Phys. 85, 2754 (1999).

${ }^{17}$ V. E. Gantmakher and Y. B. Levinson, Carrier Scattering in Metals and Semiconductors (North Holland, Amsterdam, 1987).

${ }^{18}$ Y. Yafet, in Solid State Physics, edited by F. Seitz and D. Turnbull (Academic, New York, 1963), vol. 14 . 


\section{FIGURES}

FIG. 1. (a) Schematics of the lateral QD. Confinement in the [001] direction is achieved as in the conventional inversion layers, while the lateral confinement is provided by the electrodes (black boxes). (b) Schematic illustration of energy levels in lateral QDs. (0) and (1) mark the different states of lateral confinement. Valley-split + and - states are further split by the magnetic field into spin-up and spin-down states. The solid (dashed) arrows show electron transitions under one-phonon (two-phonon) relaxation.

FIG. 2. Dependence of one-phonon (solid lines) and two-phonon (dashed lines) spin relaxation rates on resonance frequency for temperatures of $0.5 \mathrm{~K}, 1 \mathrm{~K}$, and $2 \mathrm{~K}$.

FIG. 3. Dependence of the spin relaxation rates on resonance frequency for the case where the ratio of temperature and Zeeman splitting $t$ is kept constant. The one-phonon rates for the considered values of this ratio cannot be resolved on this scale and is presented by one curve. 


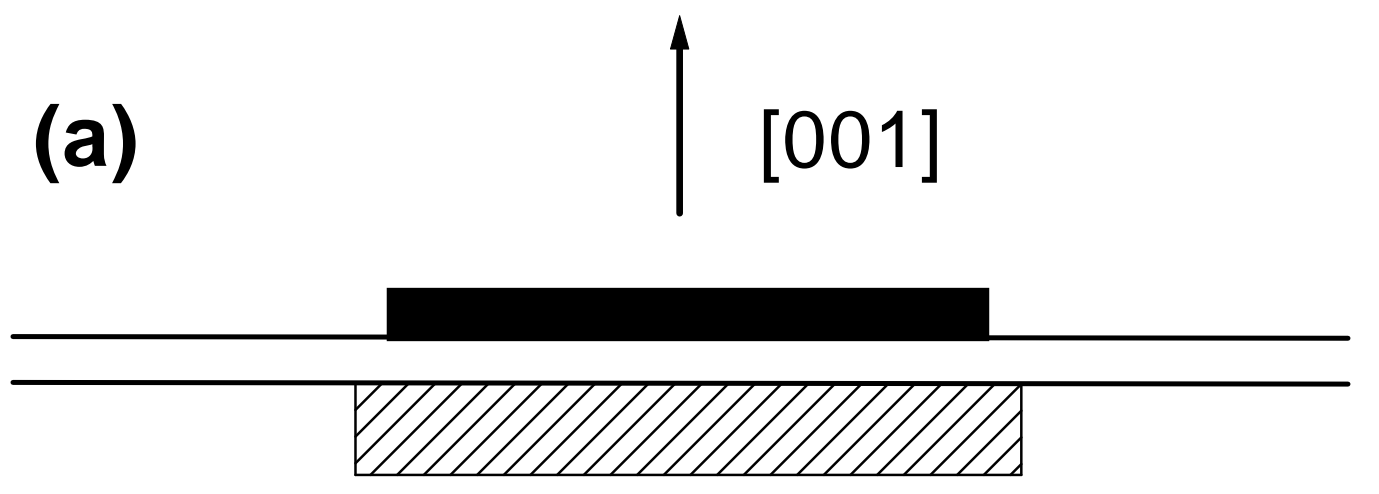

(b)

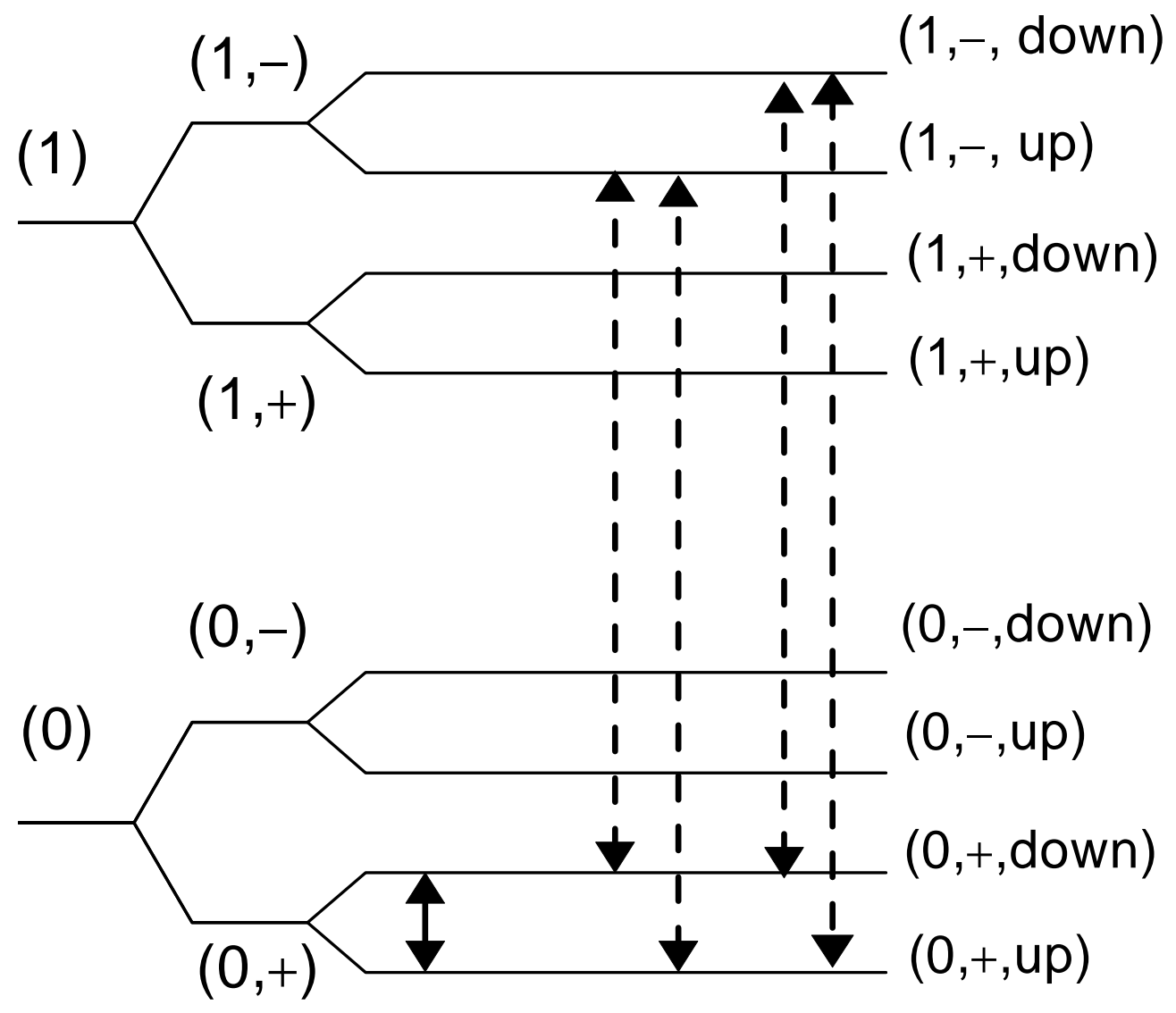

Fig.1. Glavin and Kim, PRB 


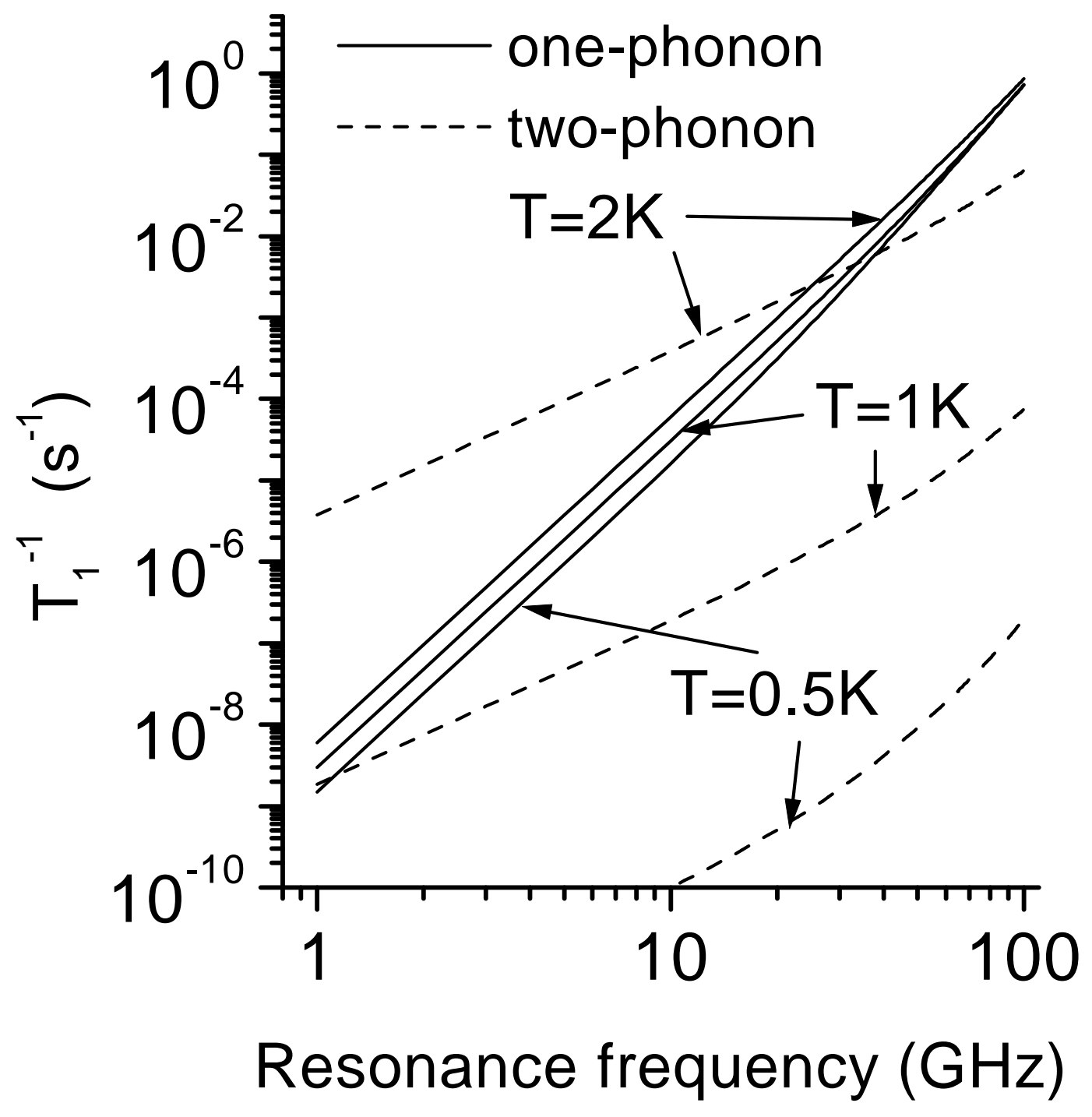

Fig.2, Glavin and Kim, PRB 


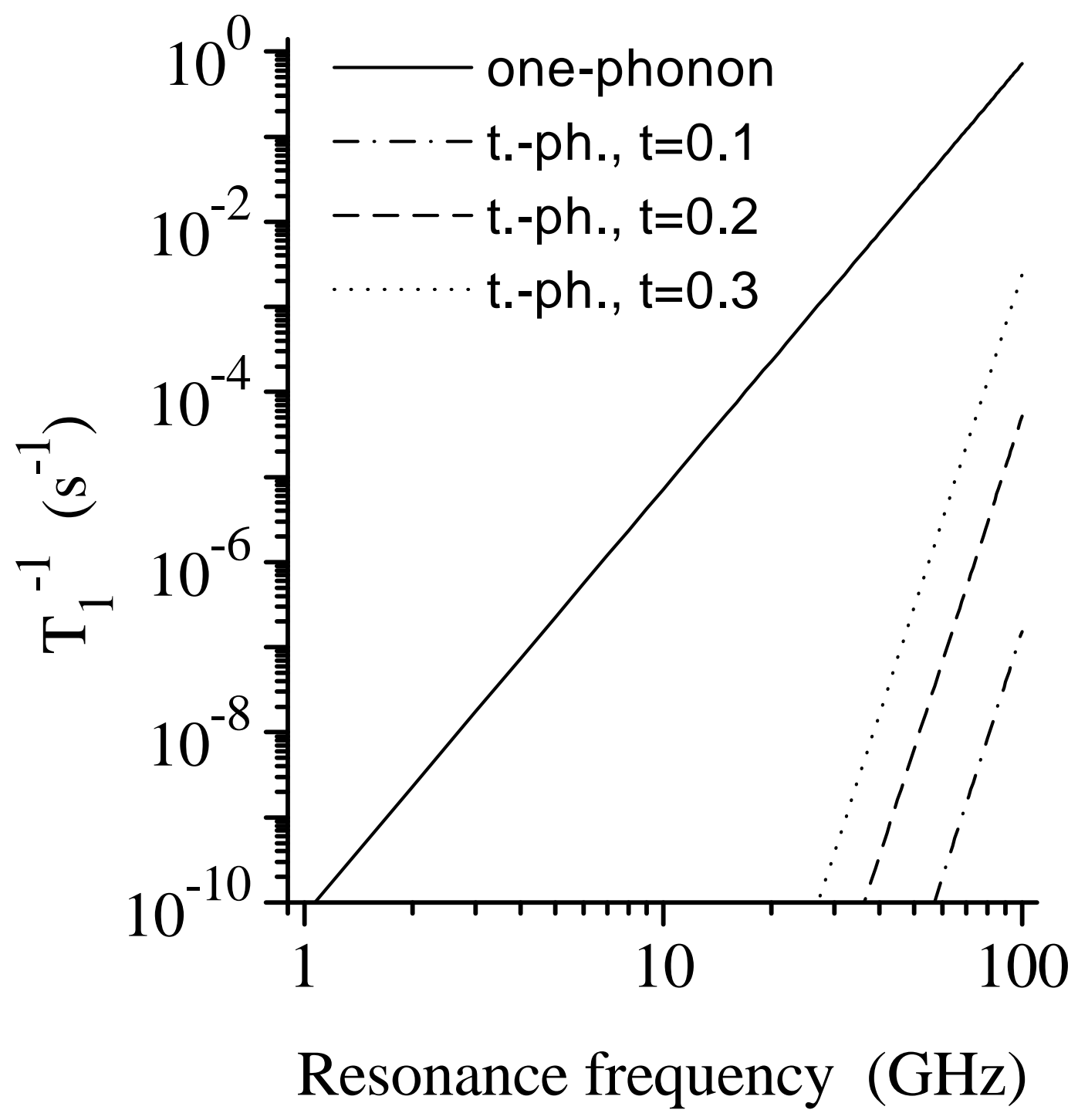

Fig.3, Glavin and Kim, PRB 